\title{
Low mass enhanced probability of pion in hadronic matter due to its Landau cut contributions
}

\author{
Sabyasachi Ghosh \\ Instituto de Fisica Teorica, Universidade Estadual Paulista, Rua Dr. Bento Teobaldo Ferraz, 271, \\ 01140-070 Sao Paulo, SP, Brazil
}

\begin{abstract}
In the real-time thermal field theory, the pion self-energy at finite temperature and density is evaluated where the different mesonic and baryonic loops are considered. The interactions of pion with the other mesons and baryons in the medium are governed by the effective hadronic Lagrangian densities whose effective strength of coupling constants have been determined from the experimental decay widths of the mesons and baryons. The detail branch cut structures of these different mesonic and baryonic loops are analyzed. The Landau cut contributions of different baryon and meson loops become only relevant around the pion pole and it is completely appeared in presence of medium. The in-medium spectral function of pion has been plotted for different values of temperature, baryon chemical potential as well as three momentum of the pion. A noticeable low mass probability in pion spectral function promise to contribute in the low mass dilepton enhancement via indirect modification of $\rho$ self-energy for $\pi \pi$ loop.
\end{abstract}

\section{Introduction}

The recent reviews [1, 2] claim that the calculations based on hadronic many-body theory are very successful to describe the low mass dimuon enhancement measured by the NA60 collaboration [3], where a strong broadening of rho meson are essentially concluded as a main reason. There are two sources which are generally believed to be responsible for this broadening of the $\rho$ meson. One is coming from the direct interactions of $\rho$ with the other thermalized mesonic [4, 5, and baryonic [6, 7, 8] constituents of the medium. Another is originating from the indirect interaction of $\rho$ with medium via pion cloud, which may be modified through interactions with the surrounding medium [9, 10, 11. The latter source indicates that the in-medium modification of pion may create an effect on $\rho$ meson propagation in the medium, for which the rate of low mass dileptons are expected to be enhanced. In this context, the present manuscript is intended to investigate the in-medium modification of pion propagation due to strong interaction with the other thermalized mesons and baryons in the medium. For this purpose the in-medium pion self-energy for different mesonic and baryonic loops are calculated in the real-time thermal field theory (RTF) and their detailed branch cut structures are explicitly analyzed. With respect to earlier investigations [12, 13, 14, 15, 16, 17, 18, one of the major contribution of this present article is that an extensive set of baryonic loops are taken to estimate the in-medium self-energy of pion. The $\pi \sigma$ and $\pi \rho$ loops have been taken as mesonic loops because the resonances $\sigma$ and $\rho$ have to be considered in effective hadronic model to reproduced the experimental data of $\pi \pi$ scattering cross section [19].

The manuscript is organized as follows. The expression of thermal propagator for $\pi$ meson is explicitly derived in the next section and then the detail calculations of pion self-energy at finite temperature and density are addressed in Sec. (3). The baryon and meson loop calculations are 
separately discussed in two subsection of the Sec. (3). The detailed numerical results are discussed in Sec. (4) and at last section the intention of the article is summarized.

\section{Pion propagator in the medium}

In the real-time formulation of thermal field theory, any two point function like propagator acquires $2 \times 2$ matrix structure. The free propagator matrix of pion at finite temperature can be defined as

$$
D_{a b}(k)=i \int d^{4} x e^{i k x}\left\langle T_{c} \pi(x) \pi(0)\right\rangle_{a b}
$$

where the subscripts $a, b(=1,2)$ are thermal indices, $\pi(x)$ is pion field and $T_{c}$ denotes time ordering with respect to a symmetrical contour in the plane of the complex time variable [20]. The notation $\langle\mathcal{O}\rangle$ is used to represent ensemble average of any operator $\mathcal{O},\langle\mathcal{O}\rangle=\operatorname{Tr} \frac{e^{\beta H} \mathcal{O}}{\operatorname{Tr} e^{\beta H}}$, where $\operatorname{Tr}$ indicates trace over a complete set of states. With the help of the Dyson equation, the complete in-medium $\pi$-propagator can be expressed as

$$
D_{a b}(k)=D_{a b}^{(0)}(k)-D_{a c}^{(0)}(k) \Pi_{c d}(k) D_{d b}(k)
$$

where $D_{a b}^{(0)}(k)$ and $\Pi_{a b}(k)$ are free propagator and self-energy matrices of pion at finite temperature. By the diagonalization procedure [21] the thermal indices $(a, b, c, d)$ can be removed and it gives the following equation :

$$
\bar{D}(k)=\bar{D}^{(0)}(k)-\bar{D}^{(0)}(k) \bar{\Pi}(k) \bar{D}(k)
$$

where the quantities with bars represent the corresponding diagonal components. The diagonal element of free propagator is expressed as

$$
\bar{D}^{(0)}(k)=\frac{-1}{k^{2}-m_{\pi}^{2}+i \eta}
$$

which is exactly similar with the vacuum free propagator. Using Eq. (44) in (3) we get the diagonal element of complete pion propagator,

$$
\bar{D}(k)=\frac{-1}{k^{2}-m_{\pi}^{2}-\bar{\Pi}(k)}
$$

whose imaginary part provides the Breit-Wigner type structure of pion spectral function,

$$
A_{\pi}(k)=\operatorname{Im} \bar{D}(k)=\frac{-\operatorname{Im} \bar{\Pi}(k)}{\left\{k^{2}-m_{\pi}^{2}-\operatorname{Re} \bar{\Pi}(k)\right\}^{2}+\{\operatorname{Im} \bar{\Pi}(k)\}^{2}} .
$$

Here the information of medium is embedded in the imaginary and real part of the pion self-energy ( $\operatorname{Im} \bar{\Pi}$ and $\operatorname{Re} \bar{\Pi})$ which will be derived in next subsection.

\section{Pion self-energy in the medium}

In this section, the different meson and baryon loop diagrams (shown in Fig. 1) of $\pi$ meson, have been evaluated in order to investigate the in-medium modification of pion in hadronic matter. The calculations of meson and baryon loops are separately addressed below. 


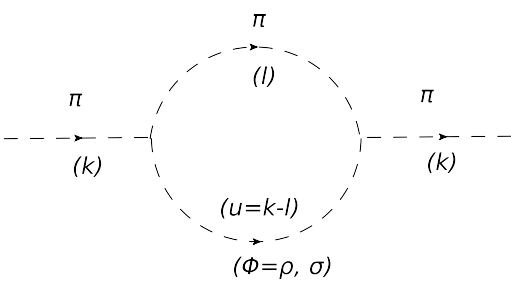

(a)

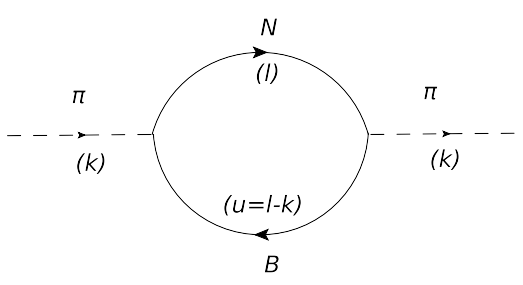

(b)

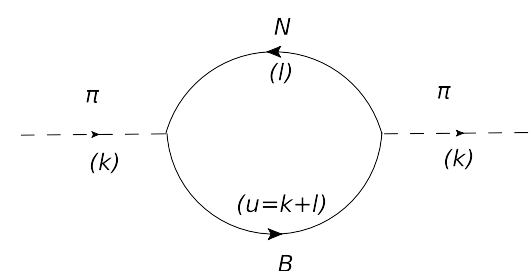

(c)

Figure 1: Self-energy diagrams of pion for meson loops $(\pi \Phi)$ is shown in diagram (a). The baryon loops $(N B)$ of pion self-energy can be represented in two possible ways - (b) and (c).

\subsection{Meson loops}

From the lowest order $\chi \mathrm{PT}$, the estimated $\pi \pi$ cross section in free space is well in agreement with the experimental data up to the center-of-mass energy $\sqrt{s}=0.5 \mathrm{GeV}$. Beyond this value of $\sqrt{s}$, the $\sigma$ and $\rho$ resonances play an important role to explain the experimental data. By the unitarization technique, the $\sigma$ and $\rho$ resonances can be generated dynamically [22] in the amplitude. An alternative way [19, 23], which is followed in the present paper, is to incorporate these resonances by using the effective Lagrangian densities for $\pi \pi \sigma$ and $\pi \pi \rho$ interactions:

$$
\mathcal{L}=g_{\rho} \vec{\rho}_{\mu} \cdot \vec{\pi} \times \partial^{\mu} \vec{\pi}+\frac{g_{\sigma}}{2} m_{\sigma} \vec{\pi} \cdot \vec{\pi} \sigma,
$$

where the coupling constants $\left(g_{\sigma}=5.82\right.$ and $\left.g_{\rho}=6\right)$ are fixed from their experimental decay widths of $\sigma$ and $\rho$ mesons in their $\pi \pi$ channels [23. Serious defect of phenomenological interactions is that they often do not respect constraints from chiral symmetry. Owing to the appearance of $\sigma$ and $\rho$ resonances in $\pi \pi$ scattering cross section, we have considered $\pi \sigma$ and $\pi \rho$ loops to calculate inmedium pion self-energy where the interaction part is governed by the effective Lagrangian densities, mentioned in Eq. (7). This pion self-energy function for $\pi \sigma$ and $\pi \rho$ loops has diagrammatically been represented by the Fig[1(a). The 11-component of pion self energy for the $\pi \Phi$ loop (where $\Phi$ denotes $\sigma$ or $\rho$ in generalized way) is given by

$$
\Pi_{M}^{11}(k)=i \int \frac{d^{4} l}{(2 \pi)^{4}} L(k, l) D^{11}\left(l, m_{\pi}\right) D^{11}\left(k-l, m_{\Phi}\right)
$$

where the vertex factors,

$$
L(k, l)=-\frac{g_{\sigma}^{2} m_{\sigma}^{2}}{4}
$$

for the $\pi \sigma$ loop, and

$$
L(k, l)=-\frac{g_{\rho}^{2}}{m_{\rho}^{2}}\left\{k^{2}\left(k^{2}-m_{\rho}^{2}\right)+l^{2}\left(l^{2}-m_{\rho}^{2}\right)-2\left[(k \cdot l) m_{\rho}^{2}+k^{2} l^{2}\right]\right\},
$$

for the $\pi \rho$ loop have been obtained from the effective hadronic Lagrangian, given in Eq. (7). The $D^{11}\left(l, m_{\pi}\right)$ and $D^{11}\left(k-l, m_{\Phi}\right)$ in (8) are the 11-component of the scalar propagator for $\pi$ and $\Phi$ meson respectively. For example,

$$
D^{11}\left(l, m_{\pi}\right)=\frac{-1}{l^{2}-m_{\pi}^{2}+i \eta}+2 i \pi n_{l} \delta\left(l^{2}-m_{\pi}^{2}\right)
$$


where $n_{l}=\frac{1}{e^{\beta \omega_{l}}-1}$ is Bose-Einstein distribution function of pion with its on-shell energy, $\omega_{l}=$ $\sqrt{\overrightarrow{l^{2}}+m_{\pi}^{2}}$. Similar to the propagator matrix, the self-energy matrix can also be diagonalized into a single component and the diagonal element and 11 component are related as [21, 24]

$$
\begin{aligned}
\operatorname{Im} \bar{\Pi}_{M}(k) & =\tanh \left(\frac{\beta k_{0}}{2}\right) \operatorname{Im} \Pi_{M}^{11}(k) \\
\operatorname{Re} \bar{\Pi}_{M}(k) & =\operatorname{Re} \Pi_{M}^{11}(k) .
\end{aligned}
$$

After doing the $l_{0}$ integration of Eq. (8) and then using the Eq. (12), the diagonal element of the pion self-energy matrix is obtained as [24]

$$
\begin{aligned}
\bar{\Pi}_{M}(k)= & \int \frac{d^{3} l}{(2 \pi)^{3}} \frac{1}{4 \omega_{l} \omega_{u}}\left[\frac{\left(1+n_{l}\right) L_{1}+n_{u} L_{3}}{k_{0}-\omega_{l}-\omega_{u}+i \eta \epsilon\left(k_{0}\right)}+\frac{-n_{l} L_{1}+n_{u} L_{4}}{k_{0}-\omega_{l}+\omega_{u}+i \eta \epsilon\left(k_{0}\right)}\right. \\
& \left.+\frac{n_{l} L_{2}-n_{u} L_{3}}{k_{0}+\omega_{l}-\omega_{u}+i \eta \epsilon\left(k_{0}\right)}+\frac{-n_{l} L_{2}-\left(1+n_{u}\right) L_{4}}{k_{0}+\omega_{l}+\omega_{u}+i \eta \epsilon\left(k_{0}\right)}\right]
\end{aligned}
$$

where $L_{i}, i=1, . .4$ denote the values of $L\left(l_{0}\right)$ for $l_{0}=\omega_{l},-\omega_{l}, k_{0}-\omega_{u}, k_{0}+\omega_{u}$ respectively and $n_{u}=\frac{1}{e^{\beta \omega_{u}-1}}$ is Bose-Einstein distribution function for $\Phi$ resonance with its on-shell energy, $\omega_{u}=$ $\sqrt{(\vec{k}-\vec{l})^{2}+m_{\Phi}^{2}}$. The imaginary part in the relevant Landau and unitary cut regions are respectively given below

$$
\operatorname{Im} \bar{\Pi}_{M}=-\frac{\epsilon\left(q_{0}\right)}{16 \pi|\vec{k}|} \int_{\widetilde{\omega}_{l}^{+}}^{\widetilde{\omega}_{l}^{-}} d \widetilde{\omega}_{l} L_{2}\left\{n\left(\widetilde{\omega}_{l}\right)-n\left(\widetilde{\omega}_{u}=k_{0}+\widetilde{\omega}_{l}\right)\right\}
$$

and

$$
\operatorname{Im} \bar{\Pi}_{M}=-\frac{\epsilon\left(q_{0}\right)}{16 \pi|\vec{k}|} \int_{\omega_{l}^{-}}^{\omega_{l}^{+}} d \omega_{l} L_{1}\left\{1+n\left(\omega_{l}\right)+n\left(k_{0}-\omega_{l}\right)\right\}
$$

where the integration limits $\omega_{l}^{ \pm}=\frac{S_{\pi}^{2}}{2 k^{2}}\left(k_{0} \pm|\vec{k}| W_{\pi}\right), \widetilde{\omega}_{l}^{ \pm}=\frac{S_{\pi}^{2}}{2 k^{2}}\left(-k_{0} \pm|\vec{k}| W_{\pi}\right)$ with $W_{\pi}=\sqrt{1-\frac{4 k^{2} m_{\pi}^{2}}{S_{\pi}^{4}}}$ and $S_{\pi}^{2}=k^{2}-m_{\Phi}^{2}+m_{\pi}^{2}$. These two regions of branch cuts are $\vec{k}<k_{0}<\sqrt{\vec{k}^{2}+\left(m_{\Phi}-m_{\pi}\right)^{2}}$ and $\sqrt{\vec{k}^{2}+\left(m_{\Phi}+m_{\pi}\right)^{2}}<k_{0}<\infty$ respectively.

The real part of the self-energy can be easily read off from (13) in terms of principal value integrals and we do not write them here.

In realistic scenario, the $\sigma$ meson should not be treated as stable particle as considered in our previous framework as it exhibits a broad spectral function in vacuum. Hence, to take into account its broad width, the Eq. (13) has been convoluted as (see e.g. [23, 28, 8, 29])

$$
\bar{\Pi}_{M}\left(k, m_{\sigma}\right)=\left[\int_{\left(m_{\sigma}^{-}\right)^{2}}^{\left(m_{\sigma}^{+}\right)^{2}} d p^{2} A_{\sigma}(p) \bar{\Pi}_{M}(k, p)\right] /\left[\int_{\left(m_{\sigma}^{-}\right)^{2}}^{\left(m_{\sigma}^{+}\right)^{2}} d p^{2} A_{\sigma}(p)\right],
$$

where $\bar{\Pi}_{M}(k, p)$ is the narrow-width expression self-energy given in Eq. (13), with $m_{\sigma}$ replaced by $p ; A_{\sigma}\left(p^{2}\right)$ is the spectral density:

$$
A_{\sigma}\left(p^{2}\right)=\frac{1}{\pi} \operatorname{Im}\left[\frac{-1}{p^{2}-m_{\sigma}^{2}+i p \Gamma_{\sigma}(p)}\right] .
$$

$\Gamma_{\sigma}(p)$ is the vacuum spectral widths of the mesons:

$$
\Gamma_{\sigma}(p)=\frac{3 g_{\sigma}^{2} m_{\sigma}^{2}}{32 \pi p}\left(1-\frac{4 m_{\pi}^{2}}{p^{2}}\right)^{1 / 2}
$$

In the integration limits, $m_{\sigma}^{ \pm}=m_{\sigma} \pm 2 \Gamma_{\sigma}^{0}$, with $\Gamma_{\sigma}^{0}=\Gamma_{\sigma}\left(p=m_{\sigma}\right)$. 


\subsection{Baryon loops}

Next we will calculate the baryon loops of pion self-energy in the same framework of RTF. Besides the meson loops in the medium, the pion propagator may also be undergone via different intermediate $N B$ loops, where $B$ stands for different higher mass baryons including nucleon itself. In this work we have taken an extensive set of 4-star baryon resonances with spin one-half and threehalf. These are $N(940), \Delta(1232), N^{*}(1440), N^{*}(1520), N^{*}(1535), \Delta^{*}(1600), \Delta^{*}(1620), N^{*}(1650)$, $\Delta^{*}(1700), N^{*}(1700), N^{*}(1710), N^{*}(1720)$ where their mass (in $\mathrm{MeV}$ ) are presented inside the brackets. The direct and cross diagrams of pion self-energy for $N B$ loops have been represented in the diagram 1 (b) and (c).

The 11-component of the in-medium pion self-energy for the $N B$ loop is given by

$$
\Pi_{B}^{11}(k)=i \sum_{a=-1,+1} \int \frac{d^{4} l}{(2 \pi)^{4}} L(k, l) E^{11}\left(l, m_{N}\right) E^{11}\left(l-a k, m_{B}\right)
$$

where $E^{11}\left(l, m_{N}\right)$ and $E^{11}\left(l-a k, m_{B}\right)$ are respectively scalar part of the nucleon and baryon propagators at finite temperature. In RTF its expression is as follows :

$$
\begin{aligned}
E^{11}\left(l, m_{N}\right) & =\frac{-1}{l^{2}-m_{N}^{2}+i \eta}-2 \pi i N\left(l_{0}\right) \delta\left(l^{2}-m_{N}^{2}\right) ; \quad N\left(l_{0}\right)=n_{l}^{+} \theta\left(l_{0}\right)+n_{l}^{-} \theta\left(-l_{0}\right) \\
& =-\frac{1}{2 \omega_{l}}\left(\frac{1-n_{l}^{+}}{l_{0}-\omega_{l}+i \eta}+\frac{n_{l}^{+}}{l_{0}-\omega_{l}-i \eta}-\frac{1-n_{l}^{-}}{l_{0}+\omega_{l}-i \eta}-\frac{n_{l}^{-}}{l_{0}+\omega_{l}+i \eta}\right)
\end{aligned}
$$

where $n_{l}^{ \pm}=\frac{1}{e^{\beta\left(\omega_{l} \mp \mu_{N}\right)}+1}$ is the Fermi-Dirac distribution for energy $\omega_{l}=\sqrt{\vec{l}^{2}+m_{N}^{2}}$ and the \pm sign in the superscript of $n_{l}$ refer to nucleon and anti-nucleon respectively. Here $\mu_{N}$ is the chemical potential of nucleon which is supposed to be equal with the chemical potentials of all the baryons considered here. The two values of $a$ in (19) correspond to the direct and crossed diagrams shown in Fig. 1 (b) and (c) respectively which can be obtainable from one another by changing the sign of the external momentum $k$.

Let us first discuss diagram (b) for which $a=+1$. After integrating over $l^{0}$ in Eq. (19) and then using the similar kind of relation like Eq. (12), the diagonal element of the in-medium self energy can be expressed as

$$
\begin{aligned}
\bar{\Pi}_{B}(k)= & \int \frac{d^{3} l}{(2 \pi)^{3}} \frac{1}{4 \omega_{l} \omega_{u}}\left[\frac{\left(1-n_{l}^{+}\right) L_{1}-n_{u}^{-} L_{3}}{k_{0}-\omega_{l}-\omega_{u}+i \eta \epsilon\left(k_{0}\right)}+\frac{n_{l}^{+} L_{1}-n_{u}^{+} L_{4}}{k_{0}-\omega_{l}+\omega_{u}+i \eta \epsilon\left(k_{0}\right)}\right. \\
& \left.+\frac{-n_{l}^{-} L_{2}+n_{u}^{-} L_{3}}{k_{0}+\omega_{l}-\omega_{u}+i \eta \epsilon\left(k_{0}\right)}+\frac{n_{l}^{-} L_{2}+\left(-1+n_{u}^{+}\right) L_{4}}{k_{0}+\omega_{l}+\omega_{u}+i \eta \epsilon\left(k_{0}\right)}\right]
\end{aligned}
$$

where $n_{u}^{ \pm}$are also Fermi-Dirac distribution functions for baryon and anti-baryon with $\omega_{u}=$ $\sqrt{(\vec{l}-\vec{k})^{2}+m_{B}^{2}}$ and $L_{i}, i=1, . .4$ denote the values of $L\left(l_{0}\right)$ for $l_{0}=\omega_{l},-\omega_{l}, k_{0}-\omega_{u}, k_{0}+\omega_{u}$ respectively. For the diagram (c) in Fig. 1, a similar kind of expression like Eq. (21) can also be received just by putting $a=-1$. The imaginary part of the third term of (21) will be non-zero in the Landau region $\vec{k}<k_{0}<\sqrt{\vec{k}^{2}+\left(m_{B}-m_{N}\right)^{2}}$ and only this contribution is significant for pion spectral function because pion pole is situated in this region while unitary cuts are far from it. Adding the relevant Landau cut contributions of diagram (b) and (c), the total contribution from the baryon loops is given by

$$
\begin{array}{r}
\operatorname{Im} \bar{\Pi}_{B}\left(k_{0}, \vec{k}\right)=\frac{-\epsilon\left(k_{0}\right)}{16 \pi|\vec{k}|} \int_{\widetilde{\omega}_{l}^{+}}^{\widetilde{\omega}_{l}^{-}} d \widetilde{\omega}_{l}\left[L_{1}(a=-1)\left\{-n_{+}\left(\widetilde{\omega}_{l}\right)+n_{+}\left(\widetilde{\omega}_{u}=k_{0}+\widetilde{\omega}_{l}\right)\right\}\right. \\
\left.+L_{2}(a=+1)\left\{-n_{-}\left(\widetilde{\omega}_{l}\right)+n_{-}\left(\widetilde{\omega}_{u}=k_{0}+\widetilde{\omega}_{l}\right)\right\}\right]
\end{array}
$$


where $\widetilde{\omega}_{l}^{ \pm}=\frac{S_{N}^{2}}{2 k^{2}}\left(-k^{0} \pm|\vec{k}| W_{N}\right)$ with $W_{N}=\sqrt{1-\frac{4 k^{2} m_{N}^{2}}{S_{N}^{4}}}, S_{N}^{2}=k^{2}-m_{B}^{2}+m_{N}^{2}$. For the $B=N$ (i.e. $N N$ loop), the diagram (b) and (c) are identical, hence only one of them has to be included.

Similar to mesonic case, the real part of $\operatorname{Im} \bar{\Pi}_{B}$ can also be extracted from Eq. (22) by taking its principal value integral. The thermal part of $\operatorname{Re} \Pi_{B}$ or $\operatorname{Re} \Pi_{M}$ is the main interest to estimate the mass shift of pion, which can be reflected from the shifting of peak position in the pion spectral function.

\begin{tabular}{|c|c|c|c|c|c|}
\hline Baryons & $J_{B}^{P}$ & $I_{B}$ & $\Gamma_{\text {tot }}$ & $\Gamma_{B \rightarrow N \pi}(\mathrm{B.R})$. & $f / m_{\pi}$ \\
\hline$\Delta(1232)$ & $\frac{3}{2}^{+}$ & $3 / 2$ & 0.117 & $0.117(100 \%)$ & 15.7 \\
$N^{*}(1440)$ & $\frac{1}{2}^{+}$ & $1 / 2$ & 0.300 & $0.195(65 \%)$ & 2.5 \\
$N^{*}(1520)$ & $\frac{3}{2}^{-}$ & $1 / 2$ & 0.115 & $0.069(60 \%)$ & 11.6 \\
$N^{*}(1535)$ & $\frac{1}{2}^{-}$ & $1 / 2$ & 0.150 & $0.068(45 \%)$ & 1.14 \\
$\Delta^{*}(1600)$ & $\frac{3}{2}^{+}$ & $3 / 2$ & 0.320 & $0.054(17 \%)$ & 3.4 \\
$\Delta^{*}(1620)$ & $\frac{1}{2}^{-}$ & $3 / 2$ & 0.140 & $0.035(25 \%)$ & 1.22 \\
$N^{*}(1650)$ & $\frac{1}{2}^{-}$ & $1 / 2$ & 0.150 & $0.105(70 \%)$ & 1.14 \\
$\Delta^{*}(1700)$ & $\frac{3}{2}^{-}$ & $3 / 2$ & 0.300 & $0.045(15 \%)$ & 9.5 \\
$N^{*}(1700)$ & $\frac{3}{2}^{-}$ & $1 / 2$ & 0.100 & $0.012(12 \%)$ & 2.8 \\
$N^{*}(1710)$ & $\frac{1}{2}^{+}$ & $1 / 2$ & 0.100 & $0.012(12 \%)$ & 0.35 \\
$N^{*}(1720)$ & $\frac{3}{2}^{+}$ & $1 / 2$ & 0.250 & $0.028(11 \%)$ & 1.18 \\
\hline
\end{tabular}

Table 1: From the left to right columns, the table contain the baryons, their spin-parity quantum numbers $J_{B}^{P}$, isospin $I_{B}$, total decay width $\Gamma_{\text {tot }}$, decay width in $N \pi$ channels $\Gamma_{B \rightarrow N \pi}$ or $\Gamma_{B}$ in Eq. (26) (brackets displaying its Branching Ratio) and at the last coupling constants $f / m_{\pi}$.

To calculate $L(k, l)$ for baryon loops we have used the effective $B N \pi$ interaction Lagrangian densities [25]

$$
\begin{aligned}
\mathcal{L} & =\frac{f}{m_{\pi}} \bar{\psi}_{B} \gamma^{\mu}\left\{\begin{array}{c}
i \gamma^{5} \\
1
\end{array}\right\} \psi_{N} \partial_{\mu} \pi+\text { h.c. for } J_{B}^{P}=\frac{1}{2}^{ \pm} \\
& =\frac{f}{m_{\pi}} \bar{\psi}_{B}^{\mu}\left\{\begin{array}{c}
1 \\
i \gamma^{5}
\end{array}\right\} \psi_{N} \partial_{\mu} \pi+\text { h.c. for } J_{B}^{P}=\frac{3}{2}^{ \pm}
\end{aligned}
$$




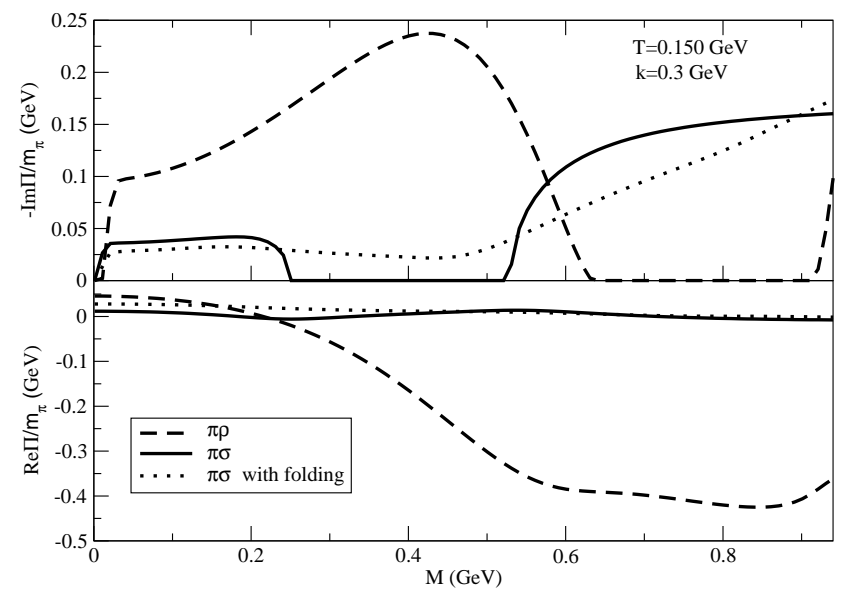

Figure 2: Imaginary (upper panel) and real (lower panel) part of pion self-energy for $\pi \sigma$ (solid line) and $\pi \rho$ (dashed line) loops. In the imaginary part of the pion self-energy, two separate regions of Landau and unitary cuts are distinctly revealed. The dotted line represents the $\pi \sigma$ loop contribution after foding by the broad vacuum spectral function of $\sigma$.

where $J_{B}$ and $P$ are spin and parity quantum numbers of corresponding baryon, $B$. The effective strength of the coupling constants $f / m_{\pi}$ for different $B N \pi$ interactions can be determined from the experimental vacuum widths of corresponding $B \rightarrow N \pi$ decays. From above Lagrangian densities, one can easily deduce

$$
\begin{aligned}
L(k, l) & =-\left(\frac{f}{m_{\pi}}\right)^{2} \operatorname{Tr}\left[\not k\left(l-a \not k-P m_{B}\right) \not k\left(l+m_{N}\right)\right] \\
& =-4\left(\frac{f}{m_{\pi}}\right)^{2}\left[2(k \cdot l)^{2}-a(k \cdot l) k^{2}-k^{2}\left(l^{2}+m_{N} m_{B}\right)\right]
\end{aligned}
$$

for $J_{B}^{P}=\frac{1}{2}^{ \pm}$and

$$
\begin{aligned}
L(k, l)= & -\left(\frac{f}{m_{\pi}}\right)^{2} \operatorname{Tr}\left[( l + m _ { N } ) ( l - a \not k + P m _ { B } ) k _ { \mu } k _ { \nu } \left\{-g^{\mu \nu}+\frac{1}{3} \gamma^{\mu} \gamma^{\nu}\right.\right. \\
& \left.\left.+\frac{2}{3 m_{B}^{2}}(l-a k)^{\mu}(l-a k)^{\nu}+\frac{1}{3 m_{B}}\left(\gamma^{\mu}(l-a k)^{\nu}-(l-a k)^{\mu} \gamma^{\nu}\right)\right\}\right] \\
= & -\frac{8}{3 m_{B}^{2}}\left(\frac{f}{m_{\pi}}\right)^{2}\left[m_{N} m_{B}+l^{2}-a(k \cdot l)\right]\left[\left(l \cdot k-a k^{2}\right)^{2}-k^{2} m_{B}^{2}\right]
\end{aligned}
$$

for $J_{B}^{P}=\frac{3}{2}^{ \pm}$. Following the Ref. [1], a soft monopole form factor $F=\left(\Lambda^{2}-m_{\pi}^{2}\right) /\left(\Lambda^{2}+\vec{k}^{2}\right)$ with $\Lambda=0.3 \mathrm{GeV}$ has been multiplied in the $\pi N B$ vertex to take into account its finite size.

The Lagrangian densities in (23) are not displaying its isospin structures. For $J_{B}^{P}=\frac{1}{2}^{ \pm}$and $J_{B}^{P}=\frac{3}{2}^{ \pm}$, these isospin structures should be $\bar{\psi} \vec{\tau} \cdot \vec{\pi} \psi$ and $\bar{\psi} \vec{T} \cdot \vec{\pi} \psi$ respectively, where $\vec{T}$ and $\vec{\tau}$ stand for the usual spin 3/2 transition and Pauli operator. These isospin structures provide appropriate isospin factors, which have to be multiplied with the expressions of corresponding $N B$ loop diagrams. The isospin factor for $N N$ or $N N^{*}$ loops is $I_{\pi \rightarrow N N, N^{*}}=2$ and for the $N \Delta$ or $N \Delta^{*}$, it is $I_{\pi \rightarrow N \Delta, \Delta^{*}}=4 / 3$.

Next we have calculated vacuum width of different baryons in the $N \pi$ decay channel to fix their corresponding coupling constants $f / m_{\pi}$. With the help of the Lagrangian densities (7), vacuum 


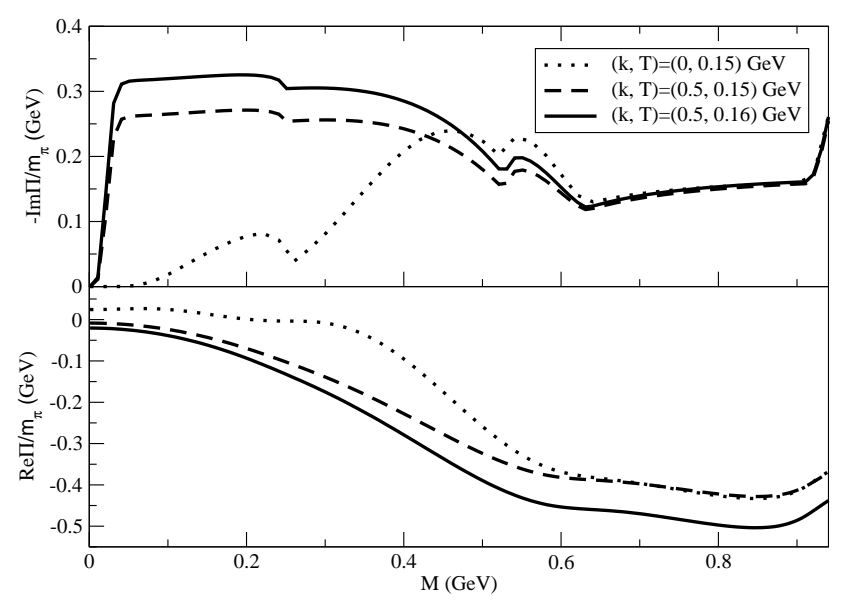

Figure 3: Imaginary (upper panel) and real (lower panel) part of total pion self-energy coming from the meson loops are presented for different set of temperature $T$ and three momentum $\vec{k}$.

decay width of baryons $B$ for $N \pi$ channel can be obtained as

$$
\begin{aligned}
\Gamma_{B} & =\frac{I_{N^{*} \rightarrow \pi N}}{2 J_{B}+1}\left(\frac{f}{m_{\pi}}\right)^{2} \frac{\left|\vec{p}_{c m}\right|}{2 \pi m_{B}}\left[2 m_{B}\left|\vec{p}_{c m}\right|^{2}+m_{\pi}^{2}\left(\omega_{N}-P m_{N}\right)\right] \text { for } J_{B}^{P}=\frac{1}{2}^{ \pm} \\
& =\frac{I_{\Delta, \Delta^{*} \rightarrow \pi N}}{2 J_{B}+1}\left(\frac{f}{m_{\pi}}\right)^{2} \frac{\left|\vec{p}_{c m}\right|^{3}}{3 \pi m_{B}}\left[\omega_{N}+P m_{N}\right] \text { for } \quad J_{B}^{P}=\frac{3}{2}^{ \pm}
\end{aligned}
$$

where $\left|\vec{p}_{c m}\right|=\frac{\sqrt{\left\{m_{B}^{2}-\left(m_{N}+m_{\pi}\right)^{2}\right\}\left\{m_{B}^{2}-\left(m_{N}-m_{\pi}\right)^{2}\right\}}}{2 m_{B}}$ and $\omega_{N}=\sqrt{\left|\vec{p}_{c m}\right|^{2}+m_{N}^{2}}$. The isospin factors are $I_{N^{*} \rightarrow \pi N}=3$ and $I_{\Delta, \Delta^{*} \rightarrow \pi N}=1$ for the $N \pi$ decay channels of $N^{*}$ and $\Delta^{*}$ (or $\Delta$ ) respectively. Putting the experimental values [26] of $\Gamma_{B}$ in Eq. (26), the values of coupling constants $f / m_{\pi}$ have been fixed, which are shown in a Table (1).

\section{Results and discussion}

Now let us discuss the results of numerical evaluation. In Fig. (2), the imaginary (upper panel) and real (lower panel) part of pion self-energy for $\pi \sigma$ (solid line) and $\pi \rho$ (dashed line) loops are plotted against the invariant mass $M$, where $M=\sqrt{k_{0}^{2}-\vec{k}^{2}}$. The Landau and unitary regions for $\pi \sigma$ and $\pi \rho$ loops are distinctly observed from the upper panel of the Fig. (2). The Landau regions of the $\pi \sigma$ and $\pi \rho$ loops are respectively $\left(M=0\right.$ to $m_{\sigma}-m_{\pi}$ i.e. 0 to $\left.0.25 \mathrm{GeV}\right)$ and $\left(M=0\right.$ to $m_{\rho}-m_{\pi}$ i.e. 0 to $0.63 \mathrm{GeV})$ whereas their unitary regions start respectively from $\left(M=m_{\sigma}+m_{\pi}=0.53\right.$ $\mathrm{GeV})$ and $\left(M=m_{\rho}+m_{\pi}=0.91 \mathrm{GeV}\right)$ to $\infty$. Since the unitary regions are far away from the pion pole $\left(M=m_{\pi}=0.14 \mathrm{GeV}\right)$, the unitary cut contributions may not play any dominating role in the spectral profile of pion. Rather the Landau cut contributions seem to be responsible for determining the spectral profile of pion. For $\pi \sigma$ loop, the broad vacuum spectral shape of $\sigma$ is taken into account by using Eq. (16) and the corresponding results are shown by dotted line in Fig. (2). From the upper panel of the Fig. (2), the dotted line indicates that due to the folding, the Landau and unitary cut regions are overlaped by each other and their contributions are also reduced. For the different set of temperature $T$ and the three momentum $\vec{k}$, the imaginary (upper panel) and real (lower panel) part of total self-energy after summing the contributions of $\pi \sigma$ and $\pi \rho$ loops have been shown in the Fig. (3). Here we see that the numerical strength of imaginary 


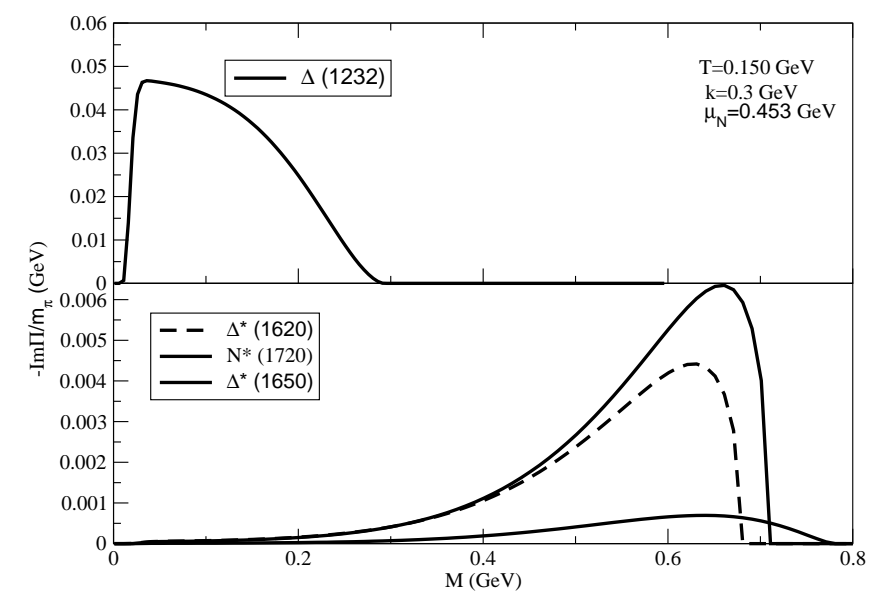

Figure 4: Imaginary part of pion self-energy for different $N B$ loops. $B=$ $\Delta^{*}(1620), N^{*}(1650), N^{*}(1720)$ are displayed in lower panel while $B=\Delta(1232)$ is shown in upper panel. we have taken the medium parameters $T=0.15 \mathrm{GeV}, \mu_{N}=0.453 \mathrm{GeV}$, for which the nucleon density become $\rho_{N}=0.55 \rho_{0}$ ( $\rho_{0}$ is saturation nuclear matter density).

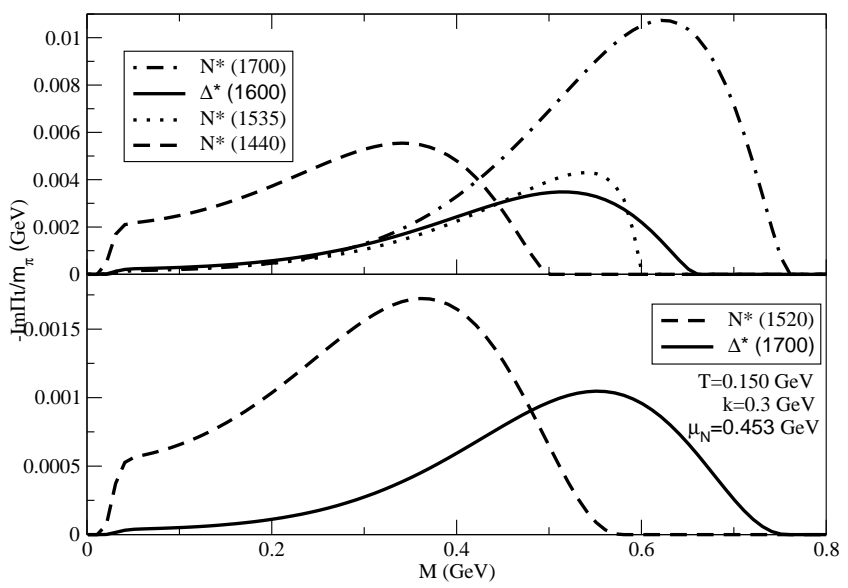

Figure 5: Same as Fig. (44) for rest of the baryons $B=N^{*}(1440), N^{*}(1535), \Delta^{*}(1600) N^{*}(1700)$ (upper panel) and $B=N^{*}(1520), \Delta^{*}(1700)$ (lower panel). 


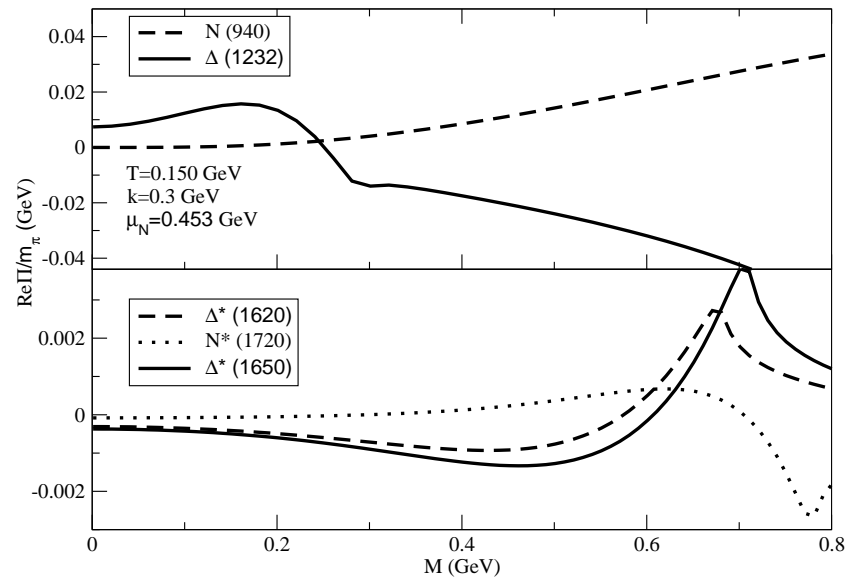

Figure 6: The corresponding results of Fig. (44) for the real part of pion self-energy. Only $N N$ loop contribution is additionally presented here which has vanishing Landau cut contribution in Fig. (4).

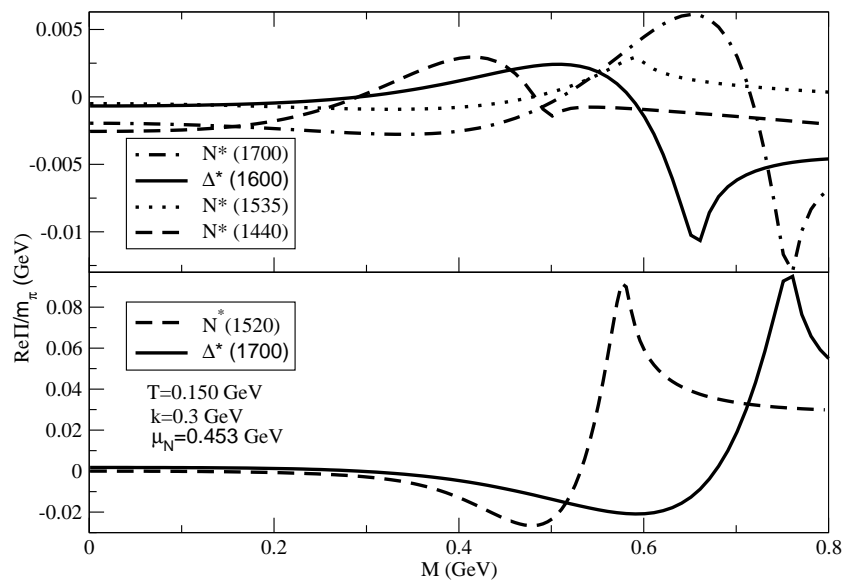

Figure 7: The corresponding results of Fig. (5) for the real part of pion self-energy.. 


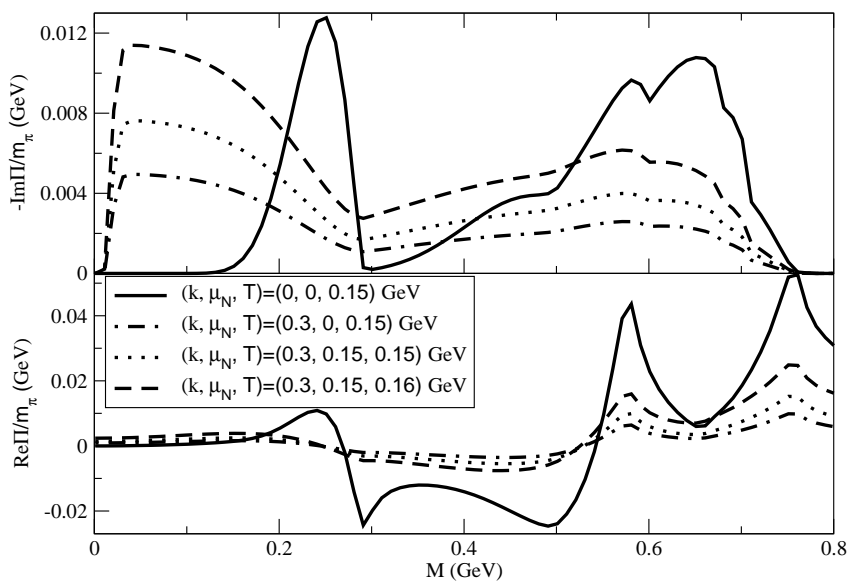

Figure 8: Imaginary (upper panel) and real (lower panel) part of total pion self-energy coming from the baryon loops are presented for different set of temperature $T$, baryon chemical potential $\mu_{N}$ and three momentum $\vec{k}$.

part of pion self-energy is significantly enhanced in the low $M$ regions (including the pion pole) for finite $\vec{k}$. It also increases with the increasing of the $\vec{k}$ and $T$. The real part of the pion self-energy become very small around it's pole position.

Next we will discuss about the results of pion self-energy which are coming from the different $N B$ loops. The Fig. (44) demonstrates the imaginary part of pion self-energy for the baryons $B=\Delta(1232)$ (upper panel) and $B=\Delta^{*}(1620), N^{*}(1650), N^{*}(1720)$ (lower panel). Whereas the results for baryons $B=N^{*}(1440), N^{*}(1535), \Delta^{*}(1600), N^{*}(1700)$ (upper panel) and $B=$ $N^{*}(1520), \Delta^{*}(1700)$ (lower panel) are displayed in the Fig. (5). The result of the $B=N^{*}(1710)$ is not revealed with the other baryons as its strength is very low. In those figures the Landau regions of different loops are clearly identified. As an example the Landau region of the $N \Delta$ loop is $\left(M=0\right.$ to $m_{\Delta}-m_{N}$ i.e. 0 to $\left.0.292 \mathrm{GeV}\right)$. Fig. (6) and (7) present the corresponding results of real part for different baryons. Summing all the baryon loops the total self-energy is represented in Fig. (8) where upper and lower panel are exhibiting the imaginary and real part respectively for different set of $\left(\vec{k}, T\right.$ and $\left.\mu_{N}\right)$. Similar to mesonic loops, $\operatorname{Im} \Pi_{B}$ in low mass region is sensitively enhanced for finite values of $\vec{k}$ and it also increases with the increasing of $T$ and $\mu_{N}$. The upper panel of Fig. (8) clearly demonstrates this fact. The non-zero values of $\operatorname{Im}_{B}(M)$ for $N B$ loops (or $\operatorname{Im} \Pi_{M}(M)$ for $\pi \Phi$ loops) in the Landau cuts, $0<M<m_{B}-m_{N}$ (or $0<M<m_{\Phi}-m_{\pi}$ ) basically interprets the statistical probability of pion due to scattering with medium constituents [27]. The possible scattering processes are as follows. During propagation in the medium, pion may disappear by absorbing a thermalized $N$ (or $\pi$ ) from the medium to create a thermalized $B$ (or $\Phi$ ). Again pion may appear by absorbing a thermalized $B$ (or $\Phi$ ) from the medium as well as by emitting a thermalized $N$ (or $\pi$ ). Hence these kind of scattering processes provide the statistical probability to pion and this probability in the low $M$ region is quite high for finite values of $\vec{k}, T$ and $\mu_{N}$. From the upper panel of Fig. (3) and (8), one can notice that the low mass probability of pion for mesonic loops is dominating over that of the baryonic loops.

To compare with some earlier results from Refs. [14, 15, 16, we have generated the numerical values of pion optical potential $\frac{\Pi\left(k_{0}=\omega_{k}, \vec{k}\right)}{2 \omega_{k}}$, where $\omega_{k}=\sqrt{\vec{k}^{2}+m_{\pi}^{2}}$. Its imaginary (upper panel) and real (lower panel) part for mesonic (solid line) and baryonic (dotted line) matter are plotted against $\vec{k}$ in Fig. (9). For mesonic matter, our imaginary part of pion potential is qualitatively similar with the results, obtained by Shuryak [14] (solid line with square) and Rapp et al. [15] 


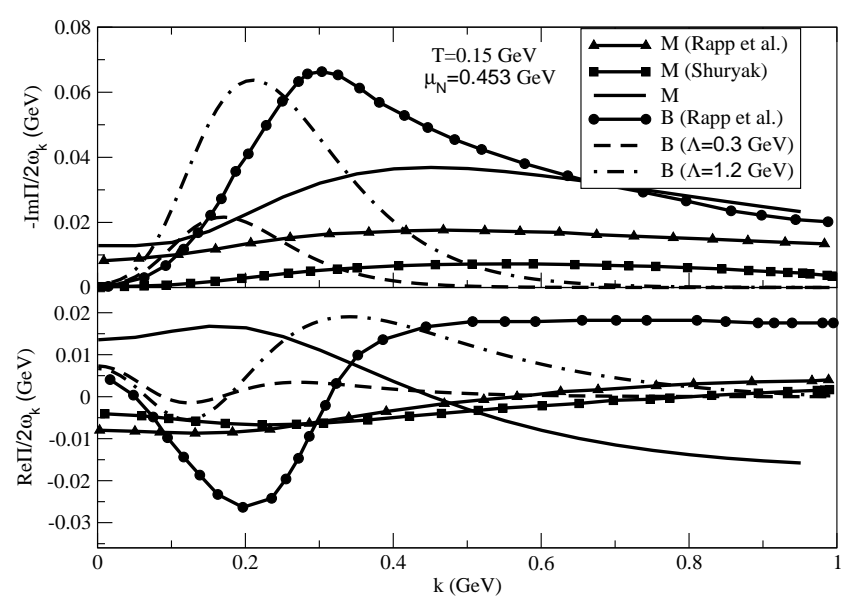

Figure 9: The imaginary (upper panel) and real part (lower panel) of pion potential for mesonic matter, obtained by Shuryak [14] (solid line with square), Rapp et al. [15] (solid line with triangle) and for baryonic matter, obtained by Rapp et al. [16] (solid line with circle). Our corresponding results for meson loops, $N \Delta$ loop at $\Lambda=0.3$ and $1.2 \mathrm{GeV}$ are shown by solid, dashed and dashdotted line respectively.

(solid line with triangle) but quantitatively larger than them. One of the reason for this quantative difference may be that our results are originated from the effective $\pi \pi \rho$ and $\pi \pi \sigma$ interactions. In baryonic matter (considering $N$ and $\Delta$ in the medium), our imaginary and real part both follow approximately similar $\vec{k}$ dependency as followed in Ref. 16 (solid line with circle). In Fig (10), We have also compared our baryonic results with the results of Tolos et al. [18, where the pion self-energy incorporates a momentum independent s-wave and a momentum dependent p-wave piece originated from the one and two nucleon-hole and delta-hole excitations, plus short-range correlations. Unlike to the results of Tolos et al. [18, our results and the results of Rapp et al. [16] exhibit a non-monotonic momentum dependence of pion optical potential with a peak structure in its imaginary part. From the Eq. (22) this nature can be grossly understood. The numerical integration of thermal distribution will roughly be close to a sine hyperbolic function of momentum, which will be a rapidly increasing function whereas the $1 / 16 \pi \vec{k}$ term before the integral will make it to be a decreasing function. Hence the net effect of these two opposite dependence of momentum create such kind of non-monotonic momentum dependent potential. In Ref. [18, incorporating the extra two loop kind of nucleon-hole and delta-hole excitations as well as an explicit function of $\Delta$ width with external variable may be one of the key reason for exhibiting the monotonically increasing behavior of pion optical potential with momentum.

Using the imaginary and real part of pion self-energy in the Eq. (6), pion spectral function can be numerically estimated. The contributions of meson and baryon loops in the pion spectral function are cumulatively shown in Fig. (11). After adding $N \Delta$ as well as the other $N B$ loops with the meson loops, the peak of the pion spectral function is gradually reduced and shifted toward the higher $M$. This fact can be noticed very well in the lower panel of Fig. (11) for the parameters $\left(T, \mu_{N}, \vec{k}\right)=(0.15,0.4,0) \mathrm{GeV}$. From the lower panel of Fig. (8), the positive values of $\operatorname{Re} \Pi_{B}$ around the pion pole already indicates the fact. By changing the three momentum $\vec{k}$ from 0 to $0.5 \mathrm{GeV}$, the peak strength of the pion spectral function has been reduced significantly, which can be observed in the upper panel of Fig. (11). The reason for such kind of reduction is because of the enhancement of Im $\Pi$ at finite $\vec{k}$, which has been observed in the upper panel of Fig. (3) and (8). This kind of softening of the pion pole for increasing momentum also observed in some earlier 


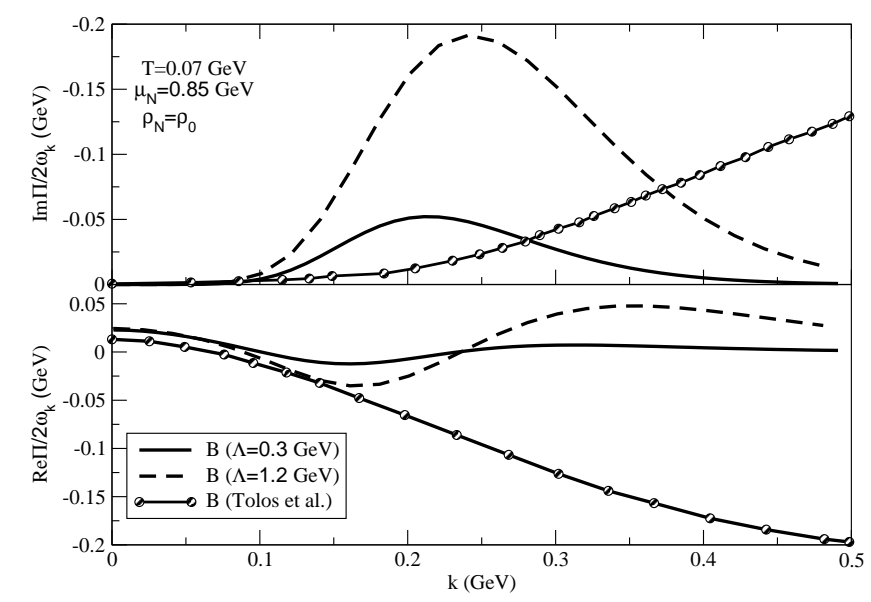

Figure 10: The imaginary (upper panel) and real part (lower panel) of pion potential at temperature, $T=0.07 \mathrm{GeV}$ and nucleon density, $\rho_{N}=\rho_{0}$. Solid (for $\Lambda=0.3 \mathrm{GeV}$ ) and dashed line (for $\Lambda=1.2$ $\mathrm{GeV}$ ) show our results for $N \Delta$ loop whereas the solid line with circle shows the corresponding results of Tolos et. al [18].

works [11, 18]. From Fig. (11), we also observe that the $N \Delta$ loop dominates over the rest of the $N B$ loops to determine the $\mu_{N}$ dependency of pion spectral profile. The Landau cut structures of the other $N B$ loops are dominantly contributed to the higher $M$ regions, which are slightly away from the pion pole. Hence, their contributions may be considered as a negligible correction part in the pion spectral function with respect to the contribution of $N \Delta$ loop. Similar dominance of $N \Delta$ loop over the other baryon loops was also noticed by Post et al. 25]. However, by increasing the pion momentum $\vec{k}$ this correction part of $\operatorname{Im} \Pi / m_{\pi}$ can be dominant over the contribution of $N \Delta$ loop as shown in the Table (2).

\begin{tabular}{|c|c|c|c|c|}
\hline$\vec{k}$ & $\operatorname{Im} \Pi / m_{\pi}(N \Delta)$ & $\operatorname{Im} \Pi / m_{\pi}(N B)$ & $\operatorname{Re} \Pi / m_{\pi}(N \Delta)$ & $\operatorname{Re} \Pi / m_{\pi}(N B)$ \\
\hline 0 & $1.4 \mathrm{MeV}$ & $1.4 \mathrm{MeV}$ & $8.4 \mathrm{MeV}$ & $11.4 \mathrm{MeV}$ \\
$300 \mathrm{MeV}$ & $38.6 \mathrm{MeV}$ & $43.5 \mathrm{MeV}$ & $15.1 \mathrm{MeV}$ & $15.9 \mathrm{MeV}$ \\
$500 \mathrm{MeV}$ & $3.3 \mathrm{MeV}$ & $10.1 \mathrm{MeV}$ & $5.6 \mathrm{MeV}$ & $3.4 \mathrm{MeV}$ \\
\hline
\end{tabular}

Table 2: Table shows imaginary and real part of pion self-energy coming from $N \Delta$ loop only and all $N B$ loops (including $N \Delta$ ) for different values of pion momentum $\vec{k}$.

Using the total pion self-energy after summing all the meson and baryon loops, we can get a full spectral function of $\pi$ meson at finite temperature and density. For different sets of $\left(T, \mu_{N}\right)$ and $\vec{k}$, this explicit structure of pion spectral function are respectively demonstrated in upper and lower panel of Fig. (12). Due to noticeable Landau cut contribution of pion in the low mass 


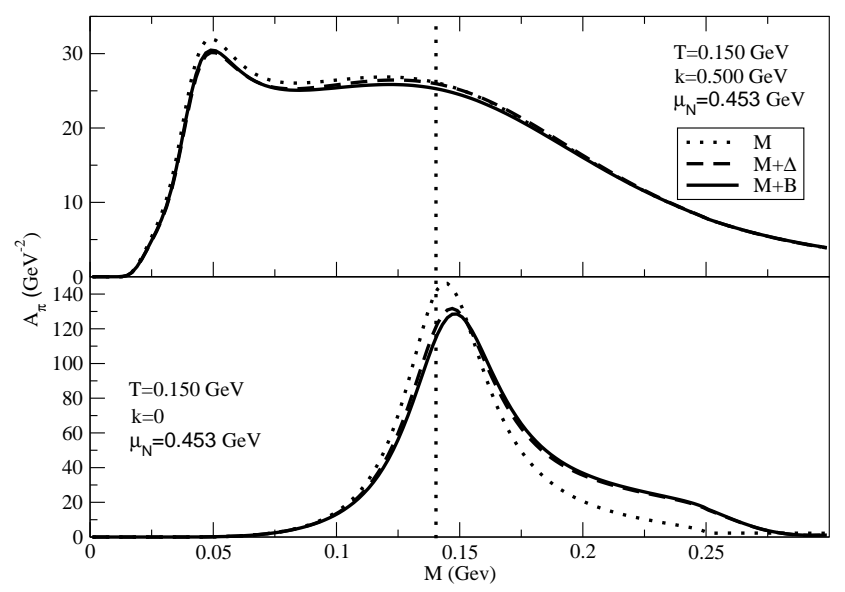

Figure 11: The contributions in pion spectral function from meson (dotted line), meson $+\Delta$ (dashed line) and meson + all baryon (solid line) loops are displayed for $\left(T, \mu_{N}, \vec{k}\right)=(0.15,0.453,0) \mathrm{GeV}$ (lower panel) and $\left(T, \mu_{N}, \vec{k}\right)=(0.15,0.453,0.5) \mathrm{GeV}$ (upper panel).

region (below its pole position), there will be some Bose enhanced probability of $\rho \rightarrow \pi \pi$ (along with $\pi \pi \rightarrow \rho$ ) below the vacuum threshold $2 m_{\pi}$, which may have some contribution in the low mass dilepton enhancement. When we will apply this explicit in-medium pion spectral function to generate dilepton spectra via $\rho$ meson modification, the probability of pion at high momentum will definitely be suppressed due to its statistical weight governed by the Bose-Einstein distribution function. Hence, a selective finite momentum range will be relevant in the dilepton spectra and the low mass enhanced probability of pion in that particular range may have some influence in the low mass dilepton spectra. This is our next interest of our future work.

\section{Summary and conclusion}

To summarize, the in-medium changes of pion propagation due to different mesonic and baryonic quantum fluctuation at finite temperature is investigated in the RTF. Since effective hadronic Lagrangian for $\pi \pi \sigma$ and $\pi \pi \rho$ interactions can alternatively describe the vacuum $\pi \pi$ scattering cross section, hence $\pi \sigma$ and $\pi \rho$ loops have been considered as relevant mesonic quantum fluctuations of pion propagation in the effective theory. The imaginary and real part of pion self-energy for those mesonic loops have been evaluated in the RTF. To account the baryonic quantum fluctuation of pion propagation at finite temperature, an extensive set of nucleon-baryon loops are taken. After summing all the mesonic and baryonic loop contributions the total self-energy of pion in hot and dense nuclear matter has been numerically estimated. The imaginary and real part of this total self-energy determine the Breit-Wigner type structure of in-medium pion spectral function, which is appeared to be slightly complex in nature because of the non-trivial branch cut structures of selfenergy function. At high temperature as well as density, the pion spectral profile are broadened with their attenuated peak structures. The main punch point of this article is the low mass probability of pion which is highly sensitive with pion's momentum $(\vec{k})$ and also with the medium parameters $\left(T\right.$ and $\left.\mu_{N}\right)$. This probability is completely appeared because of medium as Landau cuts of pion self-energy for different mesonic and baryonic loops are responsible for this contribution. Because of this low mass probability of pion, a non-zero Bose enhanced probability of $\rho \rightarrow \pi \pi$ (along with $\pi \pi \rightarrow \rho$ ) can be received below the vacuum threshold $2 m_{\pi}$, which promise to contribute in the low mass dilepton enhancement. However, a particular range of pion momentum will be responsible 


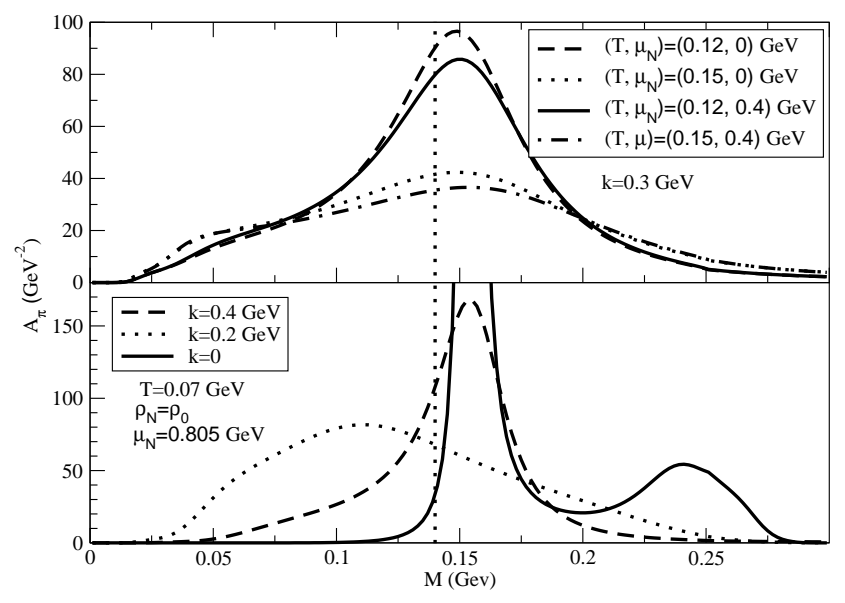

Figure 12: Full pion spectral function for different sets of $\left(T, \mu_{N}\right)$ and $\vec{k}$ are shown in upper and lower panel respectively.

for this low mass dilepton enhancement when we will apply this in-medium pion spectral function on the dilepton spectra via $\rho$ meson modification. This efforts of calculating the low mass dilepton rate, which will be coming from the $\rho$ meson self-energy for modified $\pi \pi$ loop, are in progress and will be addressed in due course.

Acknowledgment : The work is financially supported by Fundacao de Amparo a Pesquisa do Estado de Sao Paulo, FAPESP (Brazilian agencies), under Contract No. 2012/16766-0. I am very grateful to Prof. Gastao Krein for his academic and non-academic support during my postdoctoral period in Brazil.

\section{References}

[1] R. Rapp, Adv. High Energy Phys. 2013, 148253 (2013); R. Rapp, J. Wambach, Adv. Nucl. Phys. 25, 1 (2000).

[2] P. Mohanty, S. Ghosh, S. Mitra, Adv. High Energy Phys. 2013, 176578, (2013).

[3] R. Arnaldi et al. for the NA60 collaboration, Phys. Rev. Lett. 100, 022302 (2008); Eur. Phys. J. C 61, 711 (2009); S. Damjanovic et al. for the NA60 Collaboration, J. Phys. G: Nucl. Part. Phys. 35, 104036 (2008).

[4] R. Rapp, C. Gale, Phys. Rev. C 60, 024903 (1999)

[5] S. Ghosh, S. Sarkar, S. Mallik, Eur. Phys. J. C 70 (2010) 251.

[6] B. Friman and H.J. Pirner, Nucl.Phys. A 617 (1997) 496.

[7] W. Peters, M. Post, H. Lenske, S. Leupold and U. Mosel, Nucl. Phys. A 632 (1998) 109.

[8] S. Ghosh, S. Sarkar, Nucl. Phys. A 870 (2011) 94.

[9] G. Chanfray and P. Schuck, Nucl. Phys. A 555 (1993) 329.

[10] M. Herrmann, B. Friman and W. Norenberg, Nucl. Phys. A 560 (1993) 411 
[11] M. Urban, M. Buballa, R. Rapp, J. Wambach, Nucl. Phys. A 673 (2000) 357.

[12] A. Schenk, Nucl. Phys. B 363 (1991) 97.

[13] G. Chanfray, D. Davesne, Z. Phys. A 349, 65 (1994).

[14] E. V. Shuryak, Nucl. Phys. A 533 (1991) 761.

[15] R. Rapp and J. Wambach, Phys. Lett. B 315 (1993) 220.

[16] R. Rapp, J. Wambach, Nucl. Phys. A 573 (1994) 626.

[17] R. Rapp, G. Chanfray, J. Wambach, Nucl. Phys. A 617 (1997) 472.

[18] L. Tolos, A. Ramos, A. Polls, Phys. Rev. C 65, 054907 (2002).

[19] S. Mitra, S. Ghosh, S. Sarkar Phys. Rev. C 85 (2012) 064917.

[20] A.J. Niemi, G.W. Semenoff, Ann. Phys. 152, 105 (1984)

[21] R. L. Kobes and G. W. Semenoff, Nucl. Phys. 260, 714 (1985).

[22] D. Fernandez-Fraile and A. Gomez Nicola, Eur. Phys. J. C 62, 37 (2009); Int. J. Mod. Phys. E 16, 3010 (2007); Eur. Phys. J. A 31, 848 (2007).

[23] S. Ghosh, G. Krein, S. Sarkar, Phys. Rev. C 89, 045201 (2014).

[24] S. Ghosh, Probing spectral properties of hadrons in hot and dense hadronic matter (Ph.D. Thesis) HBNI (India), 2012, http://www.hbni.ac.in/phdthesis/thesis_june2013 /PHYS04200704005_Sabyasachi_Ghosh.pdf

[25] M. Post, S. Leupold, U. Mosel, Nucl. Phys. A 741, 81 (2004).

[26] J. Beringer et al. (Particle Data Group) Phys. Rev. D 86, 010001 (2012).

[27] H.A. Weldon, Phys. Rev. D 28, 2007 (1983).

[28] P. Gonzalez, E. Oset, and J. Vijande, Phys. Rev. C 79, 025209 (2009).

[29] S. Ghosh and S. Sarkar, Eur. Phys. J. A 49, 97 (2013). 